\title{
A preliminary survey of dementia amongst geriatric patients admitted to a single medical unit at the Teaching Hospital Jaffna (THJ), Sri Lanka using a locally validated scale - the concise cognitive assessment scale (CONCOG).
}

\author{
Arasalingam $A^{1}$, Suganthan $N^{1}$ \\ ${ }^{1}$ Department of Medicine, Faculty of Medicine, University of Jaffna, Sri Lanka. \\ Corresponded Author: Arasalingam, A. \\ Email: ajini.arasalingam@gmail.com \\ https://orcid.org/0000-0001-9321-6238
}

\begin{abstract}
:
Testing cognition is the background to dementia assessment. Most available assessment batteries are not developed or validated in developing countries. The Concise Cognitive test (CONCOG) validated in an elderly urban Sri Lankan population tested orientation to time, naming, registering 4 words, copying, verbal fluency and recall of the 4 words using native vernacular, creating a culturally acceptable and contextualized assessment tool.
\end{abstract}

Patients above the age of 60 years admitted to the professorial medical unit of THJ were screened after excluding confounding factors (delirium, sepsis, chronic illness, degenerative neurological diseases) which would have affected the test results. Patients who consented voluntarily were screened using the CONCOG test. A convenient sampling method was used. The severity was decided based on the score (20 - 23 mild dementia, 13 - 19 moderate dementia and 12 or less severe dementia)

Of 1001 patients screened (mean age $73.29 \pm 5.8$ years; 522 or $52.1 \%$ males; 479 or $47.9 \%$ females) $675(67.43 \%)$ had cognitive impairment. Of those with cognitive impairment mean age was $75.41 \pm 5.69,329(48.7 \%)$ males and 346(51.3\%) females.

293/675(43.41\%) had mild dementia (mean age $71.8 \pm 3.66$; $47.1 \%$ males). 325/675 (48.15\%) had moderate dementia (mean age $77.46 \pm 4.93 ; 49.5 \%$ males). 57/675 (8.44\%) had severe dementia (mean age $82.3 \pm 6.23 ; 52.6 \%$ males $)$. Of the 1001 screened only $326(32.57 \%)$ had no evidence of dementia.

In Conclusion $91.7 \%$ of subjects with cognitive impairment had mild and moderate dementia which responds to cognitive rehabilitation and medication. Early detection should be encouraged. A larger population based screening should be carried out to conform the above findings.
(Key words: Dementia, CONCOG (concise cognitive scale), Jaffna, Northern Province.)

\section{Background:}

Sri Lanka has a rapidly ageing population and it is a well known fact that the prevalence of dementia increases with increasing age. Studies on the prevalence of dementia in Sri Lanka is sparse. Dementia is a neurodegenerative clinical syndrome seen in a variety of conditions such as Alzheimer's disease, Vascular Parkinson's disease, Lewy body dementia and many other subtypes of dementia. Dementia can also be a part of other organic diseases such as Huntington's disease, CADASIL and many others.

A study carried out in an semi-urban elderly Sinhala population in Ragama, Sri Lanka showed that there was a female preponderance amongst the demented with $61 \%$ being females of which $80 \%$ were between $65-75$ years, and the overall estimated prevalence rate was $3.98 \%$. (1) Of those diagnosed to have dementia the commonest was probable Alzheimer's disease ( $71.4 \%$ ) followed by vascular dementia(14.3\%) and these figures were higher compared to another community based study carried out in India. (1)

One of the major problems in determining the diagnosis of dementia is the fact that the tool has to be culturally contextualized and the tool should be in the vernacular of the population being tested. The Mini Mental State Examination (MMSE) in Sinhalese when evaluated against the CAMCOG (Cambridge cognitive score) had a sensitivity and specificity of $93.5 \%$ and $84.6 \%$ respectively making the Mini Mental State Score in Sinhalese a sensitive score to test for dementia in Sri Lanka. (2)

Dementia and it's early detection is currently being recognised as an important and integral part of public health, given the ever changing demographic 
pattern with an increase in elderly and consequently a greater burden on direct and indirect health cost. Studies show that only about $50 \%$ of cases with dementia are diagnosed by physicians with the barriers being attributed to failure to recognise symptoms, limited time and perceived lack of need to determine a specific diagnosis. (3) However to the contrary the recognition of dementia in the primary care will enhance the quality of life of both the care giver and the patient. The use of appropriate, contextualized screening instruments used in the vernacular of the population being screened has shown promising results. $(4,5)$

The tool used to screen for dementia should be culturally contextualised and in the vernacular of the respective poipulation. Sri Lanka is a multi cultural and multi ethnic country with Sinhala being the language of the majority and the Tamil being the next commonly spoken language with very few who will be able to use English specially in comprehending and responding to dementia screening tools where various components of the intellectual domains are assessed. A study has shown that the culturally adapted MMSE, Informant Questionnaire on Cognitive Decline in the Elderly (IQCODE) and the Clinical Dementia Rating (CDR) can be used to screen dementia in Sri Lanka (6). The IQCODE was the best with a sensitivity of $71.4 \%$ and a specificity of $82.6 \%$ when 3.5 is the cut-off, and is culturally acceptable, easy to administer and user friendly even amongst those with low level of literacy as well as in those with visually and auditory impairment. (6) The commonly used tools in the Sri Lankan clinical settings are the Mini-Mental-State Examination and the MoCA(Montreal Cognitive Assessment tool), both of which have validated Tamil and Sinhala translations. The MoCA is free, however the MMSE is copyrighted limiting it's use. The validated Sri Lankan Tamil version of the MoCA tools has shown to be effective in diagnosing mild cognitive impairment optimal cut-off score for detection of MCI was 23/24, which yielded a sensitivity and specificity of $84.7 \%$ and $76.4 \%$, respectively. (7) Further MMSE is copyrighted and cannot be assessed freely.

The Concise Cognitive Test (CONCOG) was designed to overcome the above short comings and describe the reliability measures and age, education, and gender effects. The CONCOG has a total score of 30 , and has subtests for orientation, naming, registration, free recall and recognition of four words, semantic verbal fluency and copying. (8) This study piloted a design to overcome the short comings and describe the reliability measures and age, education, and gender effects. The CONCOG has a total score of 30 , and has subtests for orientation, naming, registration, free recall and recognition of four words, semantic verbal fluency and copying (8). The CONCOG is a reliable cognitive screening measure, with ceiling effects and is less influenced by education, and offers sub-scale scores for the major cognitive domains. The CONCOG was administered and validated against the Hybrid MMSE, by the same rater in the subjects preferred language of choice (english, tamil, sinhala) on volunteering healthy community dwelling, cognitively healthy elders.

The CONCOG being proved effective and reliable, designed in a contextualised Sri Lankan perspective we planned to use this scale to screen all elderly patients above the age of 60 years presenting to the Teaching Hospital Jaffna with a view to characterise the demographic profile of patients presenting with memory impairments.

\section{Methodology:}

\section{Subjects:}

All patients above the age of 60 years admitted to the Professorial Medical Unit, Teaching Hospital Jaffna, the largest tertiary and only Teaching Hospital in the Northern Province were recruited to the study and screened for memory impairment. These patients were admitted to the unit either as self referred or health-care personal referred for medical conditions. Subjects with confounding factors (delirium, sepsis, chronic illness, degenerative neurological diseases) which would affect the test results were excluded and the screening was done after 24 hours of admission to ensure confounders were excluded and all exclusion criteria were met. Only the patients who consented voluntarily were screened using the CONCOG test. They were briefed about the study and the benefits and risks 
of agreeing in the event of having a screening value suggestive of dementia. The study period commenced in December 2017 and continued until August 2018. A convenient sampling method was used. The severity was decided based on the score (20-23 mild dementia, 13-19 moderate dementia and 12 or less severe dementia).

\section{Clinical Assessment:}

All subjects were evaluated with a history, neurological examination, cognitive assessment using the Concise Cognitive Test (CONCOG) (8). The CONCOG screens the following domains: Orientation to time (3 points), language ( 7 points), verbal episodic memory ( 8 points), visuospatial skills (4 points), and semantic verbal fluency ( 8 points). The language item includes naming of seven body parts. The memory item includes learning, delayed free recall, and multiple choice recognition of four unrelated words. Verbal fluency (animals per minute) was chosen as the executive function item. The visuospatial domain included copying of two simple diagrams (flower and diamond within a square). The total possible score on the CONCOG is 30 .

\section{Statistical Analysis:}

The statistical analysis was carried out using the statistical package for social sciences and excel. Categorical variables were described as percentages and numerical variables were described as mean averages with standard deviations.

\section{Results:}

A total of 1001 patients were screened during the study period. The salient demographic patters are represented in the tables given below.

Table 1: Demographic pattern.

\begin{tabular}{lc}
\multicolumn{1}{l}{ Total number } & $\mathbf{1 0 0 1}$ \\
Mean age & $73.29+/-5.8$ years \\
Males & $522(52.1 \%)$ \\
Females & $479(47.9 \%)$
\end{tabular}

Table 2: Demographic pattern of those with cognitive impairment based on scores on CONCOG

\begin{tabular}{ll}
$\begin{array}{l}\text { Total number with } \\
\text { cognitive impairment }\end{array}$ & $675(67.43 \%)$ \\
$\begin{array}{l}\text { Total number without } \\
\text { cognitive impairment }\end{array}$ & $326(32.57 \%)$ \\
\hline Mean age & $74.41+/ 33.66$ years \\
Males & $329(48.7 \%)$ \\
Females & $346(51.3 \%)$
\end{tabular}

Table 3: Severity of dementia amongst those with cognitive impairment based on the CONCOG scores.

\begin{tabular}{ll}
$\begin{array}{ll}\text { Total number with } \\
\text { cognitive impairment }\end{array}$ & $675(67.43 \%)$ \\
\hline Mild dementia & $293(43.41 \%)$ \\
Moderate dementia & $325(48.15 \%)$ \\
Severe dementia & $57(8.44 \%)$
\end{tabular}

Demographic data of patients with mild, moderate and severe dementia are tabulated below.

Table 4: Demographic data of patient's with mild, moderate and severe dementia.

\begin{tabular}{|c|c|c|c|c|}
\hline $\begin{array}{c}\text { Dementia } \\
\text { severity }\end{array}$ & Number & $\begin{array}{l}\text { Male } \\
(\%)\end{array}$ & $\begin{array}{c}\text { Female } \\
(\%)\end{array}$ & $\begin{array}{c}\text { Mean age } \\
\text { (years) }\end{array}$ \\
\hline $\begin{array}{l}\text { Mild } \\
\text { dementia }\end{array}$ & $293(43.41 \%)$ & $47.1 \%$ & $52.9 \%$ & $71.83+/-3.66$ \\
\hline $\begin{array}{l}\text { Moderate } \\
\text { dementia }\end{array}$ & $325(48.15 \%)$ & $49.5 \%$ & $50.5 \%$ & $77.46+/-4.93$ \\
\hline $\begin{array}{l}\text { Severe } \\
\text { dementia }\end{array}$ & $57(8.44 \%)$ & $52.6 \%$ & $47.4 \%$ & $82.3+/-6.23$ \\
\hline
\end{tabular}

Table 5: Baseline characteristics of the study population.

Total number with cognitive impairment

675

$\begin{array}{ll}\text { Mean age } & 73.29+/-5.8 \text { years } \\ \text { CONCOG total (30) } & 20.48 \\ \text { Orientation (3) } & 1.59 \\ \text { Naming (7) } & 6.8\end{array}$




$\begin{aligned} & \text { Total number with cognitive } \\ & \text { impairment }\end{aligned}$
$\begin{aligned} & \text { Copying (4) } \\ & \text { Verbal fluency (8) }\end{aligned}$
$\begin{aligned} & \text { Memory (8) (free recall or } \\ & \text { recent memory) }\end{aligned}$

\section{Discussion:}

Dementia affects older people and is a leading contributor of disability and dependence. There is a rapid increase in the ageing population in amongst the members of the developed world including China, India and latin America. (9) It is expected that by 2050 , there will be a1.25 billion (22\%) increase in those over 60 years of age and $79 \%$ will be in the less developed regions, where there is less awareness and preparedness of the health system to cope up with this demand. Of the estimated 24.3 million people with dementia in 2001, about $60 \%$ were living in low and middle-income countries. (10) and Sri Lanka also falls into this category. Caring for a patient with dementia and support needed to improve carer support, education and training are associated with substantial increased strain and economic burden on the house hold as well as the health economics of the country which are low and middle income countries. (11) Sri Lanka a rapidly developing low-middle income country shows the typical demographic pattern of an ageing population.

Our study shows $67.43 \%$ of hospitalised patients with cognitive impairment of which $8.44 \%$ have severe dementia. A similar study in a sub-urban Sinhala community in suburban Ragama shows an estimated prevalence of $3.98 \%$. however this was a community based study. (1) The severity of dementia was not assessed in the above study. The studies on the prevalence of dementia in the Jaffna district or Northern Province, either in the community or in hospital settings has not been published. Further the unique situation of a 30 year old civil war leaving the demographic pattern of this region slightly changed with the younger population migrating and a preponderance of elderly population is noted. The mean age of the subjects with cognitive impairment was $74.41+$ /
3.66 years, and the usual pattern of worsening of dementia with age was obvious in our survey (Table 4). Most of the subjects had mild to moderate dementia 618/675 (91.56\%) and in a clinical perspective these subjects will benefit best from treatment with anticholinergic drugs (donepazil or rivastigmine).

All other scales were either modified culturally and contextualised to our country and the translations were in Tamil and Sinhala. The CONCOG was designed to suit our culture and was validated against standardised scales. The CONCOG scale demonstrates orientation, verbal fluency and memory to be the most affected amongst the subjects with memory impairment.

This study has its strengths and weaknesses. The strengths of this study include

- CONCOG is a brief instrument that can be used even in a busy clinic setting / hospital setting.

- This scale has been designed, validated and proved to be sensitive and specific in our cultural set up.

- There is a validated Tamil version.

The limitations of this study includes

- The study subjects were patients admitted to the hospital for a specific illness. Thus even though precautions were taken to remove all confounders clinically, hospitalization could have had an effect on the results.

- Elderly population have the tendency to get confused in new environments and this could have been one of the contributing factors for the lower scores in orientation, verbal fluency and memory.

This study is the first hospital based study in the Jaffna district using a large study population (1001 subjects) and will form the base for further analysis of the data and also expansion of the study to involve the community should be contemplated.

\section{Conclusion:}

Based on the study results, the prevalence of dementia is higher than in similar hospital based studies in the Southern part of the country where this scale was developed and studied. Many factors 
support the psiibility of a higher prevalence of dementia in population in the war stricken region to manifest in the post war era due to lack of stimulation and poorer cognitive reserve. Further analysis on the educational level and the effect on activities of daily living should be analysed.

\section{References:}

1. de Silva HA, Gunatilake SB, Smith AD. Prevalence of dementia in a semi-urban population in Sri Lanka: report from a regional survey. Int J Geriatr Pstchaitry 2003;18(8):711-5.

2. de Silva HA, Gunatilake SB. Mini Mental State Examination in Sinhalese: a sensitive test to screen for dementia in Sri Lanka. Int J Geriatr Pstchaitry 2002;17(2):134-9.

3. Boise L, Camicioli R, Morgan DL et al. Diagnosing dementia: Perspectives of primary care physicians. Gerontologist. 1999:39:457-64. (Pub med abstract)

4. Solomon PR, Adams FA, Groccia ME. et al. Correlational analysis of five commonly used measures of mental status / functional abilities in patients with Alzheimer's disease. Alzheimer Dis Assoc Disord. 1999:13;147-50. (abstract)

5. Khachaturian AS, Gallo JJ, Bretner JC. Performance characteristics of a two stage dementia screen in a population sample. J Clinical Epidemiology. 2000; 53:531-40. (abstract)

6. Kathriarachchi ST, Sivayogan S, Jayaratna $\mathrm{SD}$, Dharmasena SR. Comparison of three instruments used in the assessment of dementia in Sri Lanka. Indian J Psychiatry. 2005;\$\&(2):109-112.
7. Coonghe PAD, Sivayokan $S$, Fonseka P, Keshavaraj A, Malhotra R, Ostbye T. Adaptation and validation of the Tamil (Sri Lanka) Version of the MoCA. (abstract presented at the Sri Lanka Association of Geriatric Medicine Academic Sessions 2017. Colombo).

8. Srinivasan S. The concise cognitive test for dementia screening: Reliability and effects of demographic variables as compared to the mini mental state examination. Neuro India, 2010; 58(5):702-7.

9. Sousa RM, Ferri CP, Acosta D, Guerra M, et al. The contribution of chronic diseases to the prevalence of dependence among older people in Latin America, China and India. BMC Geriatr 2010;10:53.

10. Ferri $\mathrm{CP}$, Prince $\mathrm{M}$, Brayne $\mathrm{C}$, Brodaty $\mathrm{H}$, Fratiglioni L, Ganguli M, et al. Global prevalence of dementia: A Delphi consensus study. Lancet 2005; 366: 2112-7.

11. Prince $M$, Brodaty $H$, Uwakwe $R$, Acosta D, Ferri CP, et.al. Strain and its correlates among carers of people with dementia in low-income and middle-income countries. A 10/66 Dementia Research Group population based survey. Int J Geriatr Psychiatry 2012; 27: 670-682.

\section{Acknowledgement:}

I would like to thank Dr. Srinivasan Srikanth (Comsultant Neurologist) who developed this scale for granting me permission to use this study tool. 